http://dx.doi.org/10.12775/szhf.2016.015

\author{
RAFA£ Michalski \\ Uniwersytet MikoŁaja Kopernika, Toruń, Polska \\ METASIS@UMK.PL
}

\title{
Bemerkungen zu Hannah Arendts Theorie der Revolution
}

\begin{abstract}
Einleitung
Der Begriff der Revolution erfreut sich heutzutage eines schlechten Rufes - man assoziiert ihn meistens mit dem Kommunismus, mit verschiedenartigen rebellischen und anarchischen Aufständen, die eine Bedrohung für die demokratische Ordnung darstellen. Die Revolution scheint aber auch ein Stichwort zu sein, das viele - in der Öffentlichkeit artikulierten und unartikulierten - politischen Probleme und Ansprüche umfasst. Die weltweite, durch die Globalisierung verursachte Krise, das Desaster der Finanzinstitutionen oder sei es auch die neueste Migrantenkrise erwecken das Bedürfnis für die radikalen, tiefgreifenden Veränderungen in dem politischen und ökonomischen System der modernen Demokratien. Verschiedene, von allen Seiten hörbaren, oft sich selbst widersprechenden Forderungen nach "etwas ganz Neuem" bedürfen einer philosophischen Klärung der Hauptbegriffe, in denen sie ihren Ausdruck finden. Arendts Werk ist in der Hinsicht nicht nur brauchbar, aber auch empfehlenswert.
\end{abstract}


Das Buch On Revolution ${ }^{1}$ (1963, Veröffentlichung der polnischen Übersetzung erst 2003) wurde in der Periode des kaltes Krieges veröffentlicht und zwar einige Jahre vor der Revolte der „1968-er Bewegung”, die langfristig kulturelle, politische und ökonomische Reformen in vielen Ländern nach sich zog. Arendt versuchte darin das vergessene, obgleich dringende Thema der revolutionären Transformation der bestehenden Staatsform zu Tage zu bringen. Trotz der Deklarationen der Autorin selbst sollten wir uns nicht davon täuschen lassen, dass sie nur einen politiktheoretischen Ansatz entwickelt hat, denn schon beim ersten Blick auf den Text wird sein evident philosophischer Charakter gezeigt. Tatsächlich geht es Arendt nicht wie den konventionellen Historikern primär um eine Darstellung des historischen Verlaufs bestimmter Revolutionen, sondern um die Gewinnung eines Idealtyps der Revolution, der für sie zwingend mit der Idee der Freiheit und Gründung sowie der Gestaltung eines neuen politischen Systems verbunden ist, das in Freiheit und aus der Freiheit heraus lebt, also - der Republik. Unter diesem Gesichtspunkt behandelt sie die beiden grossen Revolutionen am Ende des 18. Jahrhunderts ausführlich - die amerikanische sowie die französische, die zu ihrem Leidwesen historisch eine stärkere Auswirkung auf Europa als die amerikanische hatten.

Was in der Rezeption dieses Werkes am peinlichsten zu sein scheint ist, dass das Werk Über die Revolution leider keine gebührende Anerkennung bei den Historikern und Soziologen fand. Der Umgang Arendts mit den historischen Fakten und ihre weitgehende Vernachlässigung der sozialen Ursachen für die Revolution haben vor der historischen und sozialen Wissenschaft wenig Gnade gefunden. Viele Forscher heben sehr wohl gewisse Verdienste Hannah Arendts hervor, betonen ihre grosse Belesenheit und die Kraft zu gelegentlich durchdringenden Einsichten, kommen jedoch meistens zu dem strengen Schluss, ihr Buch sei für die modernen Sozialwissenschaften wenig ergiebig. Auch bei polnischen Forschern fand Über die Revolution keinen grossen Anklang - vielleicht deswegen, da man den philosophischen Kern Arendts Überlegungen übersieht oder ihn im Voraus unterschätzt. Das volle, komplexe Verständnis der Revolution erfordert doch nicht nur die Kenntnis der geschichtlichen und sozialen Tatsachen, sondern vor allem auch die detaillierte Analyse des theoretischen, begrifflichen Zusammenhangs, in dem

${ }^{1}$ H. Arendt, On Revolution, New York 1963. In diesem Artikel basiere ich auf der deutschen Fassung des Textes: H. Arendt, Über die Revolution, München 1974. Die polnische Überetzung: H. Arendt, O Rewolucji, tłum. M. Godyń, Warszawa 2003. 
sie sich ereignete, wenn auch die Revolitionären selbst unreflektiert an den Ereignissen partizipierten, indem sie das alte Machtsystem bis in die Grundfesten erschüttert haben.

In diesem Artikel haben wir nicht genug Platz, um alle Thesen Arendts und Gedankengänge zu besprechen und zu rekonstruieren, deshalb fokussieren wir uns auf einigen Problemsträngen und nicht zuletzt auf der Kritik der liberalen Ideologie, die immer noch unsere politische Wirklichkeit weitgehend bestimmt. Arendt hat nämlich den liberal-demokratischen und marxistischen Traditionen, also den beiden dominierenden politischen Traditionen der letzten 150 Jahre, eine Art Gegenrechnung aufgemacht. Beide, liberale Demokraten und Marxisten - so ihre Grundthese - haben das Drama der neuzeitlichen Revolutionen nicht verstanden, weil sie beide nicht begriffen haben, dass das eigentlich Revolutionäre in den modernen Revolutionen immer wieder ein mißlungener Versuch der Constitutio Libertatis war - ein Versuch der Errichtung eines politischen Raumes öffentlicher Freiheit, in dem die Menschen als gleiche und freie Bürger ihre gemeinsamen Angelegenheiten in die eigene Hand nehmen ${ }^{2}$. Beide, die Liberalen und Marxisten, waren gleichermaßen einem Verständnis verhaftet, welchem nach der Endzweck der Politik etwa jenseits der Politik sei - sei es die Verfolgung der individuellen Interessen, die private Wohlfahrt der Bürger oder die Etablierung einer klassenlosen Gesellschaft. Was die Marxisten betrifft, so scheint Arendts Kritik keine Zweifel zu erregen, obwohl letztens sehr interessante Versuche zum Vorschein gekommen sind, Lenin und die durch ihn initiierte Oktober-Revolution zu rehabilitieren ${ }^{3}$. Provozierender aus heutiger Sicht wirkt aber ihre Kritik an den liberalen und sozialen Demokratie moderner Industriegesellschaften. In diesem Artikel rekapitulieren wir zunächst Arendts Argumente dafür, die sie aus der Sicht ihres Politikbegriffs und der Idee des Rätesystems (der erste und zweite Abschnitt) vorträgt. Dann teilen wir uns mit einigen kritischen Bemerkungen zu diesem Thema (der dritte Abschnitt) und zum Schluss zeigen wir die Hauptherausforderungen, die Arendts Denken den gegenwärtigen Demokratietheoretikern stellt.

\footnotetext{
${ }^{2}$ H. Arendt, Über die Revolution, München 1974, S. 85, 160.

${ }^{3}$ Vgl. S. Żiżek, Die Revolution steht bevor. Dreizehn Versuche über Lenin, übers. von N. G. Schneider, Franfurt am Main 2002.
} 


\section{Die Revolution als Raum öffentlicher Freiheit}

Arendt entwickelt ihre Kategorien am Paradigma der amerikanischen Revolution, der - ihrer Sicht nach - einzigen halbwegs gelungenen Revolution der Neuzeit. In der amerikanischen Revolution wurde das, worum es dem revolutionären Volk letztlich in allen modernen Revolutionen ging, also die Konstitution eines Raumes öffentlicher Freiheit - aufgrund glücklicher (also ganz kontingenter) Umstände, der langen Tradition lokaler Selbstverwaltung und dem politischen Engagements der Gründerväter, verdankt. Und zwar darauf kommt es Arendt an - wurde hier die Freiheit nicht nur im „negativen” Sinn der Garantie allgemeiner und gleicher Grund- und Bürgerrechte, sondern im „positiven” Sinn der Etablierung eines föderativen Systems von Institutionen realisiert, in dem die Selbstregierung der Bürger - von der Ebene lokaler Selbstverwaltung bis zur Ebene der nationalen Politik hin - zu einer in Erfahrung verankerten und zugleich immer wieder in der täglichen Praxis neu erfahrbaren Wirklichkeit werden konnte 4 .

Am amerikanischen Modell entwickelt Arendt ihre Idee eines Rätesystems als eine politische Alternative zu den traditionellen liberal-demokratischen, sozial-demokratischen und marxistischen Staatskonzeptionen. In den grossen nachfolgenden Revolutionen, insbesondere der französischen und der russischen, wurde, so Arendt, die Idee des Rätesystems immer wieder spontan vom revolutionären Volk wiederentdeckt, um dann von einer zur Macht gekommenen revolutionären Elite unterdrückt zu werden - „[...] wo immer die Revolution scheinbar siegte die Ein-Partei Diktatur, also angeblich die Diktatur des Proletariats, in Wahrheit die der Berufsrevolutionäre, sich schließlich durchsetzte; und das hat noch immer bedeutet, daß die Organe und Institutionen der Revolution schließlich von den revolutionären Parteien erledigt wurden, zumeist in einem Kampf, der erheblich blutiger war als der Kampf gegen die Kontrrevolution." Einzig in Amerika blieben die Elemente jener Tradition lokaler Selbstregierung erhalten, von der die amerikanische Revolution einst ausgegangen war. Für Arendt ist dabei wichtig, dass die Erinnerungen an das „öffentliche Glück” des Zusammenhandelns Freier und Gleicher, wie es einst ebenso in den townships ${ }^{6}$ und wards (Stadt-

\footnotetext{
${ }^{4}$ H. Arendt, Über die Revolution..., op. cit., S. 183-231.

${ }^{5}$ Ibidem, S. 338.

${ }^{6}$ „Die Stadt- und Dorfgemeinden amerikanischer Art waren in Europa schlechthin unbekannt und, vom Standpunkt eines europäischen Beobachters gesehen, brach die amerikani-
} 
räten) der vorrevolutionären Epoche, wie auch in der Phase der Konstituierung der amerikanischen Republik gewesen war, erfahrbar geworden sind. Freilich, so Arendt, wurden auch in Amerika schon bald nach der Revolution die Tendenzen zur Etablierung eines Parteienstaates und damit letztlich zur Entwicklung einer modernen Massendemokratie immer stärker, wobei, Arendt nach, in den modernen Massendemokratien die Bürger tendenziell nur noch im „negativen” Sinn frei sind, weil sie ihre politische Freiheit - die Freiheit einer in gemeinsamen Handeln und gemeinsamen Deliberieren sich verwirklichenden Selbstregierung - an ihre Repräsentanten, an Großparteien und Volksvertretungen verloren haben ${ }^{7}$. Was dem modernen Parteienstaat und der Einparteiendiktatur der sozialistischen Staaten gemeinsam ist, ist gemäß Arendt die Tendenz zur Auflösung der Politik in eine Verwaltung; die marxistische Parteidiktatur zieht gewissermaßen die Konsequenzen aus einer in der Entwicklung des liberal-demokratischen Parteiensystems angelegten politischen Entmündigung der Bürger, einer Entpolitisierung des Politischen. Arendt sah darin eine tödliche Bedrohung von der Freiheit in der modernen Welt.

Diese negativen Prozesse scheinen überall wirksam zu sein. Insofern bewährt sich Arendts Diagnose auch heute noch. Das heißt, die Idee politischer Freiheit, wie sie Arendt dem common sense der modernen Demokratien entgegenhält, hat im etablierten politischen Diskurs der Moderne keinen rechten Ort, so dass in der Erinnerung und im Denken der Menschen die entscheidenden revolutionären Ereignisse der Neuzeit nur noch verzerrt wahrgenommen werden. Arendts Neudeutung der modernen Revolutionsgeschichte und ihre Kritik an der Freiheits- und Politikvergessenheit der liberal-demokratischen Tradition ist in einem philosophischen Sinne radikal: Arendt wirft ihren Zeitgenossen (und auch uns) einen radikalen Bruch hinsichtlich der zentralen Kategorien vor, in denen die modernen Gesellschaften sich politisch artikuliert haben.

Die wichtigsten Kategorien in diesem Zusammenhang sind - Arendt nach - die miteinander streng verbundenen Begriffe des Handelns und der Freiheit. In Vita activa wird das Handeln (griech. praxis) als die höchste, für die Bildung einer humanen Welt gänzlich unverzichtbare Tätigkeit definiert ${ }^{8}$.

sche Revolution mit ihrer Lehre von Volkssouverenität in den townships aus und nahm von dorther den Staat in Besitz." Ibidem, S. 215.

${ }^{7}$ Ibidem, S. 339

${ }^{8}$ H. Arendt, Vita activa oder vom tätigen Leben, München 1981. 
Die Praxis, soweit sie der Gründung und Erhaltung politischer Gemeinwesen dient, schafft die Bedingungen für eine Kontinuität der Generationen, für die Erinnerung und damit auch für die Geschichte. Sie spielt sich zwischen den Individuen ab und zeigt gleichzeitig die Einzigartigkeit, die Verschiedenheit und die Pluralität der Menschen auf. In diesem Sinne bildet die Praxis einen Raum für die Freiheit des Menschen. Das Handeln besteht in politischer Interaktion, welche für Arendt fundamental ist. Kommunikation, d. h. „Finden des rechten Wortes im rechten Augenblick" ist bereits ein echtes, freies Handeln: „stumm ist nur die Gewalt, und schon aus diesem Grunde kann die schiere Gewalt niemals Anspruch auf Größe machen ".9. Die Freiheit der handelnden Menschen findet im öffentlichen Raum statt. Am klarsten realisiert wurde dies für Arendt in der griechischen Polis, wo das Arbeiten im privaten Raum des Haushaltes - mit allen Folgen einer Zwangsherrschaft - stattfand, während sich das Handeln im öffentlichen Raum auf der Agora abspielte. Dieser öffentliche Platz war der Ort einer politischen Kommunikation, Gestaltung und Freiheit unter Gleichen. Damit können sich Freie und Gleiche in öffentlicher Rede und Widerrede um das Wohl ihrer Gemeinschaft sorgen und bemühen. Das Handeln ist in seinen Ergebnissen nicht vorausberechenbar, denn es ist spontan und frei, was die Einzigartigkeit und die Anfälligkeit jeder Revolution für Krisen erklärt. Ebenso wie mit der Geburt eines jeden Menschen, so auch mit der Revolution, ergibt sich die Chance für einen Neuanfang. Darum gilt es - wie Arendt immer wieder betont - diesem freien Handeln möglichst weite Geltung in einem Gemeinwesen zu verschaffen.

Diese Idee politischer Freiheit war zwar in den Revolutionen der Neuzeit mehr oder weniger untergründig wirksam, aber sie „überkreuzt[e]” sich gleichsam immer schon mit dem mainstream des neuzeitlichen politischen Denkens. Faszinierend ist nun, dass Arendt ihre Kritik an der Politikvergessenheit der Moderne an zwei verschiedenen Enden der Philosophiegeschichte ansetzt: ihre wichtigsten Bezugspunkte sind Aristoteles und Heidegger, obwohl nur der erste explizit in Erscheinung tritt. Vielleicht sollte man besser sagen: ihr wichtigster Bezugspunkt ist ein mit und zugleich gegen Heidegger neu gelesener Aristoteles ${ }^{10}$.

Mit dem Autor der Nikomachischen Ethik setzt sie die Rationalität des praktischen Deliberierens, der politischen Klugheit und der politischen Ur-

\footnotetext{
${ }^{9}$ Ibidem, S. 36.

${ }^{10} \mathrm{Zu}$ Arendts kritischer Heidegger-Lektüre vgl. R. Jaeggi, Welt und Person. Zum anthropologischen Hintergrund der Gesellschaftskritik Hannah Arendts, Berlin 1997, S. 49-71.
} 
teilskraft der Rationalität des wissenschaftlichen Erkennens, des technischen Herstellens und des ökonomischen Administrierens, sowie die öffentliche Sphäre der Isonomia (Gleicheit) der privaten Sphäre der Arbeit (griech. ponos) und des individuellen Glückstreben entgegen. In allen diesen Entgegensetzungen klingen evident Aristotelische Unterscheidungen. So betrachtet, versucht Arendt eine antike, aber in der Neuzeit weitgehend verschüttete Tradition des politischen Denkens wieder gegenwärtig zu machen. Sie ist aber nicht einfach eine Neo-Aristotelikerin, denn ihr Rückgriff auf Aristoteles hat - bei aller erkennbaren Stoßrichtung gegen die „Anti-Politik” Heideggers - doch unverkennbar gewisse Züge eines Heideggerischen Umdenkens der Aristotelischen Kategorien. Arendt beschreibt Constitutio Libertatis als eine Eröffnung einer Welt, als Bruch mit dem Kontinuum der Geschichte, als radikalen Neubeginn und sie bestreitet jeden Zusammenhang zwischen den politischen Freiheitsimpulsen der Moderne und dem MenschenrechtsUniversalismus der liberalen Tradition. Arendts Kritik an der Geschichtsphilosophie und an der Fortschrittsidee sind Elemente einer Philosophie, in deren Licht die Konstitution eines Raums öffentlicher Freiheit gleichsam als ein kontingenter Akt der Menschen erscheint. Einer Menschheit, die sich dazu entschliesst, als Freie und Gleiche zusammenzuhandeln, und in derer Licht die Ausdehnung jenes Raumes politischer Freiheit als notwendigerweise begrenzt und „lokal” erscheint, als „eingezäunt”, wie Arendt selbst sagt „wie immer Freiheit je als eine greifbar weltliche Realität existiert hat, war sie räumlich begrenzt" ${ }^{\prime 1}$. Der Raum öffentlicher Freiheit ist ein wesentlich endlicher Raum, gleichsam dem „Aufscheinen eines Lichts”, in dem das Leben des Menschen in geschichtlichen Augenblicken „erglänzt” und sich zu einer Welt gemeinsamen Handelns eröffnet. Am Ende des Revolutionsbuch erinnert Arendt an den Sophokleischen Theseus, durch dessen Mund Sophokles verkündet, „woran diese Menschen sich hielten, um von der Trauer des Lebendigen nicht übermannt zu werden und aus der Finsternis der Kreatur in die Helle des Menschlichen zu gelangen. Es war Polis, der eingezäunte Raum der freien Tat und des lebendigen Wortes, die das »das Leben aufglänzen machte - ton bion lampròn poiesthai" ${ }^{12}$.

${ }^{11}$ H. Arendt, Über die Revolution..., op. cit., S. 354.

${ }^{12}$ Ibidem, S. 362. 
2. Die Revolution als nicht erfüllte Utopie der Freiheit - das Rätesystem

Über die Revolution ist in entscheidenden Zügen ein radikal un-Kantisches Buch, denn es wir ein Versuch unternommen, den für Kant und die liberale Tradition grundlegenden Zusammenhang zwischen der Idee der politischen Freiheit und dem Universalismus der Menschenrechte zu annullieren. Es ist nicht so, dass Arendt diesen Universalismus der Menschenrechte bestreitet, aber in auffalender Übereinstimmung mit ihrem Antipoden Carl Schmitt bestreitet sie die politische Relevanz dieses Universalismus: er wird zu einer nur noch moralischen Kategorie. Was die liberalen Grund- und Bürgerrechte betrifft, die in der liberalen Tradition durchaus auf der Idee der Menschenrechte fundiert waren, so versteht Arendt sie lediglich als notwendige Voraussetzungen politischer Freiheit. Als verhängnisvoll beurteilt sie deswegen die Verwechslung einer verfassungsmässigen Garantie von Grund- und Bürgerrechten mit dem, was sie die Konstitution politischer Freiheit nennt ${ }^{13}$.

Die negativen Freiheiten des modernen Verfassungsstaates ${ }^{14}$ dürfen, Arendt nach, noch universalistisch gedacht werden, aber Gleiches gilt nicht mehr für die positive Freiheiten, das heißt die politische Freiheit einer republikanischen Staatsform. Arendt setzt geradezu die letztere, die politische Freiheit, vielmehr der universalistischen Grammatik des modernen liberalen Diskurses entgegen. Es gibt freilich immer wieder Stellen im Revolutionsbuch, an denen Spuren einer universalistischen Deutung der Idee politischer Freiheit zu sehen sind. Es sind Spuren daher, da an ihnen auch der revolutionäre Universalismus der französischen und russischen Revolution in Arendts Denken sichtbar wird. Ein Beispiel ist der eben zitierte Schluß des Buches, aber deutlicher heißt es an anderer Stelle: „Was von den furchtbaren Katastrophen der Revolutionen des zwanzigsten Jahrhunderts verdeckt worden ist, ist nicht mehr und nicht weniger als diese erste, nun wahrhaft revolutionäre Hoffnung der europäischen und schließlich vielleicht aller Völker

\footnotetext{
${ }^{13}$ Ibidem, S. 185.

${ }^{14}$ In negativen Freiheitsrechten wird festgelegt, was den Staatsorganen nicht erlaubt ist: so darf der Staat weder Menschen ohne Grund und ohne Gerichtsverfahren einsperren, noch Zensur ausüben, noch in die „mediale Freiheit” (der Fall der ACTA-Abkommen) der Menschen eingreifen usw. Die von der Verfassung garantierten Freiheiten müssen natürlich für alle Individuen gleichermaßen gelten und alle Menschen müssen vor dem Gesetz gleich sein. Die negative Freiheit vom Staat ist übrigens ein Individualrecht, kann also nur von Individuen in Anspruch genommen werden. Arendts Freiheitsbegriff bezieht sich dagegen auf die öffentliche Verhältnisse.
} 
der Erde auf eine neue Staatsform, die es jedem inmitten der Massengesellschaften doch erlauben könnte, an den öffentlichen Angelegenheiten der Zeit teilzunehmen"15. Wenige Seiten später schlägt Arendt einen großen „,ideengeschichtlichen Bogen" von der amerikanischen Revolution über die französische Rätesysteme von 1789 und 1871 und die russische Rätebewegung von 1917 bis zu den Ansätzen eines Rätesystems in der ungarischen Revolution von 1956 und bemerkt: „Wieder wie im Falle der zahllosen Bünde, Cosoziationen und Konföderationen, die wir aus der Frühgeschichte Nordamerikas kennen, setzte sich das föderative Prinzip des Zusammenschlußes und Bündnisses getrennter Einheiten aus den Elementarbedingungen des Handelns durch [...]"16. Was Arendt hier sagt, ist nicht mehr und nicht weniger als das, dass die Idee der politischen Freiheit - in einem praktischen Sinne - in den „Elementarbedingungen des Handelns” angelegt ist. Was bedeutet diese Formulierung? Das Ausmaß in dem - und die Formen in denen - die politische Freiheit an Realität gewinnen kann, hängen, Arendt nach, von historischen Kontingenzen ab: von kulturellen Traditionen, von materiellen Bedingungen, ebenso wie von der Initiative, Phantasie, der Entschlossenheit und der Urteilskraft der Individuen, die diesen Raum öffentlichen Freiheit jeweilig bilden. Übrigens müssen sich die Institutionen der öffentlichen Freiheit immer auch gegenüber den partikularen Interessen des Kapitals, den Machtansprüchen politischer Eliten und der Logik des administrativen Systems bewähren und behaupten. Auf diese Weise denkt Arendt den wesentlich „lokalen” und partikularen Charakter jedes Raumes öffentlicher Freiheit zusammen mit der Möglichkeit, dass die revolutionäre Hoffnung „vielleicht alle Völker der Erde” ergreifen könnte. Dies ist Arendts eigentümliche Version eines revolutionären Universalismus, wobei zunächst unklar bleibt, wie sich dieser gleichsam anthropologisch fundierte Universalismus zum Universalismus der liberalen Menschen- und Bürgerrechte verhält.

Bevor wir im dritten Abschnitt auf diese Frage zurückkommen, möchte ich Arendts Entgegensetzung von Parteienstaat und Rätesystem, von parlamentarischen und direkter Demokratie - eine Entgegensetzung, durch welche sie zugleich ihren Begriff politischer Freiheit von dem der liberal-demokratischen Tradition abzusetzen versucht - etwas genauer betrachten.

Für Arendt gehört die Frage der Volksvertretung zu den beunruhigendsten Problemen der modernen Politik. Traditionell unterscheidet man zwi-

\footnotetext{
${ }^{15}$ H. Arendt, Über die Revolution..., op. cit., S. 341.

${ }^{16}$ Ibidem, S. 344.
} 
schen einer Repräsentation als Ersatz für die direkte Anteilnahme des Volkes und einer Volksvertretung, in der die Abgeordneten, die keineswegs stellvertretende, aber reale Regierungsmacht darstellen. Die gewählten Vertreter heutzutage am meisten die Experten - kümmern sich nicht um die öffentlichen Angelegenheiten, sondern um die partikularen Interessen ihrer Wähler (der Korporationen, der wirtschaftlichen Lobby, etc.) oder ihrer Partei. Die Vertreter werden für begrenzte Zeit zu legitimen Herrschern des Volkes. Die „Volksvertretung” bedeutet in diesem Fall, dass die Wähler periodisch zusammentreten (eigentlich nur bei der Wahlen), um sich ihrer eigenen Macht $\mathrm{zu}$ entledigen. Die parlamentarische Demokratie beruht somit wesentlich darauf, dass „[der] Staat und [die] Regierung in eine bürokratische Administration entartet, in welcher es einen eigentlich öffentlichen Raum nicht mehr gibt; selbst das Zusammenkommen der Abgeordneten wird ihn nicht mehr konstituieren, da es sich dabei ja lediglich darum handelt, bestimmten von vitalen Notwendigkeiten diktierten Interessen gerecht zu werden, und dies wird am besten von Experten entschieden. Von einem eigentlichen Raum, [...] in dem es freie Meinungsbildung und freie Wahl gibt, kann nicht mehr die Rede sein"17.

Die infolge einer Revolution spontan geborenen Räte bildeten - wie Arendt betont - von Anfang an eine tödliche Gefahr für das Parteiensystem. Sie waren nicht nur Kampforgane, sondern funktionierten auch als eine Keimzelle einer neuen staatlichen Ordnung. Das Hauptmerkmal der Räte war „,... das Streben nach einer möglichst unmittelbaren, weitgehenden und unbeschränkten Teilnahme des Einzelnen am öffentlichen Leben"18. Dies ist eine wichtige, sei es auch kontroverse These Arendts, die aus ihrer Überzeugung folgt, dass die Räte im scharfen Gegensatz zu allen revolutionären Parteigruppen, erheblich mehr an der rein politischen Frage der Staatsform interessiert waren als an der sozialen Frage. Als Beispiel führt Arendt das Budapester Arbeiterrat an, dessen Mitglieder sich ausschließlich mit politischen Fragen befassen wollten, d.h. sich an die Stelle des Staatsapparats setzen wollten. Wir könnten dazu unsere polnische "Solidarität” (Unabhängige Selbstverwaltete Gewerkschaft „Solidarität”), die sich in einer Volksbewegung gegen das herrschende kommunistische Regime wandte, hinzufügen. Freilich wurde die große Streikwelle durch rein materielle, soziale Forderungen und Bedürfnisse des Volkes, und strenggenommen durch die Preiserhöhun-

\footnotetext{
${ }^{17}$ Ibidem, S. 304.

${ }^{18}$ Ibidem, S. 338-339.
} 
gen von Fleisch, ausgelöst, aber im Laufe der Ereignisse zeigte es sich, dass die wichtigste Rolle in dieser Bewegung der strikt politischen Forderungen zukam. Deswegen scheint die These von Arendt trotz der soziologischen Kritik an ihrem Ansatz legitim zu sein. Ähnlich wie bei anderen Revolutionen wurden auch hier die spontan entstandenen Organe der Selbstverwaltung durch das Parteiensystem abgelöst. Diese Ersetzung, also die Etablierung des angeblich demokratischen Vielparteiensystems bedeutet für Arendt keineswegs, dass dadurch Verhältnisse und Institutionen geschaffen wurden, in denen der Bürger wirklich an öffentlichen Angelegenheiten teilnehmen kann. Die von Neoliberalen besungene politische Pluralität hat tatsächlich kaum mehr geleistet, als eine beschränkte Kontrolle (der Meinungs-, Presse-, Medien-, Versammlungsfreiheit, Anhörungen, Volksabstimmung, alle vier Jahre stattgefundenen Wahlen, etc.) der Regierenden durch die Regierten. Fraglos ist, dass die Parteien mit ihrem Monopol der Nominierung derer, die zur Wahl gestellt wurden, nicht mehr als Organe der Volksmacht anzusehen sind, sondern vielmehr als sehr wirksame Hilfsmittel, durch welche eben diese Macht des Volkes eingeschränkt und kontrolliert wird. Arendt bringt sogar die These vor, dass das Repräsentativsystem der modernen Demokratien sich in eine Art Oligarchie verwandelt habe. „Demokratisch” seien die Regierungsformen in der Tat, denn sie bezwecken die Volkswohlfahrt und sie dienen dem privaten Wohlbefinden. Zu den öffentlichen Angelegenheiten gehören also heutzutage vor allem soziale Fragen und ihre Besorgung liegt in den Händen von der oligarchisch konstituierten und von den Parteien selektierten Gruppe.

Der oben skizzierte Gedankengang Arendts führt zum Schluss, dass in allen Revolutionen, vor allem des zwanzigsten Jahrhunderts, die entscheidende Rolle der Konflikt zwischen den Repräsentation und dem echten politischen Handeln spielte. Was dabei überraschend zu sein scheint, ist ihr ganz unkritisches Plädoyer für die immer wieder rettende Kraft des Rätesystems, also ihre utopische Hoffnung auf eine spontane Revolution, die eine neue Staatsform ins Leben rufen könnte. Lassen wir die Philosophin selbst das Wort ergreifen, um ihren Enthusiasmus für die revolutionäre Selbstverwaltung ans Tageslicht ohne versteckte Unterstellungen zu bringen. „Öffentliche Freiheit, öffentliches Glück und die Verantwortlichkeit für öffentliche Angelegenheiten würden dann Wenigen zufallen, die in allen Gesellschafts- und Berufsschichten daran Geschmack finden. Sie sind ohnehin die politische Elite eines Landes, und kein Staat kann behaupten, seine Aufgabe zu erfüllen, keine Staatsform den Anspruch stellen, eine wirkliche Republik zu sein, die sich 
ihrer nicht bedient und ihr nicht den ihr gehörigen öffentlichen Raum zur Verfügung stellt. Vielleicht würde eine solche im wahrsten Sinne des Wortes "aristokratische« Staatsform dann nicht mehr zu dem Mittel der allgemeinen Wahlen greifen, denn nur diejenigen, die freiwillige Mitglieder einer "Elementarrepublik« sind, hätten den Beweis dafür erbracht, daß es ihnen um anderes und vielleicht um mehr geht als um ihr privates Wohlbefinden und um ihre legitimen Privatinteressen" ${ }^{19}$. Die revolutionäre - sei es proletarische oder intellektuelle oder irgendwelche andere - Elite wird keine von oben oder von unten kommende Legitimation ihrer Macht brauchen, denn sie wird eine neue Regierungsform aufgrund der Autorität bilden, die sie in der politischen Praxis erlangen wird. Wie Arendt schreibt: „Zweifellos würde diese Staatsform in voller Entfaltung wieder die uralte Gestalt der Pyramide annehmen, also die Gestalt aller Staatsformen, die wesentlich auf Autorität beruhen. Während aber in autoritären Regierungen, wie wir sie aus der Geschichte kennen, die autoritätsgebende Macht von oben nach unten "fließt«, würde in diesem Fall die Autorität weder oben noch unten ihre Quelle haben, sondern auf jeder Stufe der Pyramide gleichsam neu entstehen. Was immerhin die Richtung anzeigen mag, in welcher die Lösung eines der ernstesten politischen Probleme der Gegenwart, wie man nämlich nicht Freiheit und Gleichheit, sondern Gleichheit und Autorität vereinen kann, zu suchen ist"20.

Diese eigentümliche Begeisterung für ein Rätesystem und die scharfe Entgegensetzung von parlamentarischer und direkter Demokratie, wenn man sie wörtlich versteht, scheint naiv zu sein, weil sich die politischen Institutionen komplexer moderner Gesellschaften nicht nach dem simplen Modell eines Rätesystems neu konstruieren lassen - es sei denn, dass Arendt ernsthaft an die Auflösung des bestehenden politischen Systems denkt. Wenn nicht, klingen dann ihre Ideen wie anarchische Schwärmereien. Sie beantwortet aber solch einen potentiellen Einwand, indem sie argumentiert, dass ihre Kritiker unbegründeterweise die Regierung mit dem Zwang identifizieren. Die echte politische Macht hat aber wesentlich nichts mit der Gewalt zu tun. Überdies sind alle Revolutionen im Gegensatz zu anarchistischen Forderungen nicht staats-, regierungs-, und ordnungsfeindlich, sondern bezwecken im Gegenteil die Neugründung eines Staates und die Errichtung einer neuen Ordnung.

Sowieso können wir den Begriff des Rätesystems vorerst als Metapher für ein Netzwerk von autonomen oder teilautonomen Institutionen, Organi-

\footnotetext{
${ }^{19}$ Ibidem, S. 359-360.

${ }^{20}$ Ibidem, S. 358.
} 
sationen und Assoziationen betrachten, in denen jeweils so etwas wie eine Selbstregierung von Freien und Gleichen - und zwar in Anbetracht unterschiedlicher Hinsichten, mit unterschiedlichen Aufgabenstellungen und mit unterschiedlichen Formen der Rekrutierung von Mitgliedern - funktionieren kann. Es scheint, dass Arendt in diesem Begriff eigentlich die politischen Institutionen eines föderativen politischen Systems und ein zivilgesellschaftliches Netzwerk von autonomen und teilautonomen Assoziationen und Organisationen gemeint haben muss. Ein Zusammenhandeln Freier und Gleicher ist in der Selbstverwaltung von Universitäten oder der Selbstorganisation von verschiedenen Bürgerinitiativen im Prinzip ebenso möglich wie in den eigentlich politischen (im demokratisch-liberalen Sinne) Institutionen eines föderativen Systems. Der bedeutende Gedanke Arendts, der eigentlich erst in der Debatte zwischen den Liberalen und Kommunitaristen wieder ernsthaft aufgegriffen worden ist, ist, dass die politische Freiheit eigentlich nur im Sinne eines Systems von Institutionen der Selbstregierung erklärt werden kann und zwar folgendermaßen: die Freiheit beginnt und muss dort erfahrbar werden, wo die "gemeinsamen Angelegenheiten” den Beteiligten gleichsam noch sinnlich greifbar sind (wie in einem „eingezäunten” Raum der griechischen Polis) und als ihre eigenen unmittelbaren Angelegenheiten in autonomer Weise verhandelt werden können. Es liegt auf der Hand, dass die politische Freiheit so verstanden, etwas anderes ist und etwas mehr ist als nur die verfassungsmäßige Garantie von Grund- und Bürgerrechten. Diese sind, wie Arendt richtig bemerkt, eine Voraussetzung der Freiheit, aber nicht die Freiheit selbst.

\section{Das Problematische an dem Politikbegriff von Arendt}

Zunächst fassen wir die wichtigsten Ideen Arendts zum Thema Politik zusammen. Zu diesem Zweck müssen wir uns auf andere Texte, vor allem auf Vita activa oder Vom tätigen Leben und Zwischen Vergangenheit und Zukunft stützen. Eine der Hauptintentionen ihrer Philosophie ist sicherlich der Versuch der Wiedergewinnung eines Konzepts des Politischen als Gegenbegriff des modernen Sinnes von Politik. Sie fundiert ihren Begriff auf einer existentialistisch geprägten Anthropologie der „Pluralität” und der „Natalität”. Dieser zufolge ist die „Pluralität”, die auf der ,ursprüngliche[n] Verschiedenheit" aller Menschen voneinander beruht ${ }^{21}$, die grundlegende Voraussetzung

${ }^{21}$ H. Arendt, Vita activa..., op. cit., S. 10. 
der Politik. Aus der „Natalität”, dem „Faktum des Geborenwerdens”, leitet sie die menschliche Fähigkeit dazu ab „einen Anfang zu machen”. Politik ist also für Arendt der Bereich, in dem Menschen ihre Möglichkeit, frei zu handeln und sich von anderen zu unterscheiden, entfalten können. Beide Fähigkeiten brauchen einen Erscheinungsraum, in dem sie sich darstellen können. So wird erst die Existenz einer politisch-öffentlichen Sphäre zur Realisierung von sowohl der Pluralität als auch der Freiheit menschlichen Handelns ermöglicht. Das ist als eine Anthropologie des Politischen zu verstehen, soll aber nicht bedeuten, dass Arendt von unzerstörbaren "Natureigenschaften” des Menschen ausgeht. Im Gegenteil, sie spricht eben von der ständigen Bedrohtheit des Politischen und wendet sich gegen die essentialistische Darstellung des Menschen als einem „zoon politikon”, denn sie interpretiert das Politische als eine Form des Zusammenlebens, welche geschichtlich selten realisiert worden ist. Es sei „[...] keineswegs eine Selbstverständlichkeit und findet sich keineswegs überall da, wo Menschen zusammenleben." ${ }^{.22}$ Deshalb bezeichnet die Pluralität sowie Natalität nicht eine essentielle „Natur” des Menschen, sondern eine ständig bedrohte Möglichkeit. Die Pluralität kann schwinden (z.B. sie kann der Konformität weichen), die Freiheit kann vernichtet werden.

Arendt entwickelt einen in jeder Hinsicht unüblichen Begriff des Politischen, der sich in wesentlichen Merkmalen von dem unterscheidet, was man als Politik versteht, wenn man darunter die politische Organisationsform einer Gesellschaft begreift. Im politischen Handeln realisiert sich für sie vornehmlich die Fähigkeit, „einen Anfang zu machen”. Das „gute” kann man im Gegensatz zum bloßen Leben so als „außeralltägliches” im Gegensatz zum „alltäglichen” Leben erklären. Im Sinne Heideggers verstanden: das Politische wird zum Bereich der „Authentizität”. Das, was man z.B. mit Hobbes als primäre Aufgabe der Politik verstehen kann, also um die latenten Konflikte um soziale und materielle Ressourcen zu verhindern, um den Einzelnen Schutz und Sicherheit zu bieten und die Grundinstitutionen des bürgerlichen Lebens zu garantieren, ist für Arendt kein Genuin der politischen Aufgabe. Die echte Politik setzt einerseits die prinzipielle Existenz politischer Konflikte voraus, andererseits aber ist der Kampf um die Ressourcen nicht die Ursache für diese Konflikte. Das Politische ist nicht das Mittel, sondern der Zweck in sich selbst. Es geht also in der Politik nicht um den kompromissgestützten Ausgleich der konkurrierenden Interessen, sondern um einen gewaltlosen

\footnotetext{
${ }^{22}$ Ibidem, S. 38.
} 
Streit der Freien und Gleichen, die um das Gemeinwohl kämpfen. Damit hat Arendt ein Konfliktmodell im Sinne, ein agonales Modell des Politischen: das Politische als eine unaufhebbare Sphäre des Wettstreites, in der sich die immer wieder erneuernde radikale Verschiedenartigkeit der Personen zum Ausdruck kommt. Gestritten wird hier um die Gestaltung des gemeinsamen Lebens und der "gemeinsamen Welt”, für die es weder eine endgültige noch eine richtige Version gibt.

Man kann sich jetzt der Frage nähern, was die spezifische Logik des Politischen ausmacht. Genuin „politische” Fragen sind für Arendt solche, für die es keine „externen”, also nichtpolitischen Kriterien gibt, die anders als eben „politisch” nicht entschieden werden können. „Im Kulturellen und im Politischen, also im gesamten Bereich des öffentlichen Lebens, geht es weder um Erkenntnis, noch um Wahrheit, sondern um Urteilen und Entscheiden, um das urteilende Begutachten und Bereden der gemeinsamen Welt und die Entscheidung darüber, wie sie weiterhin aussehen und auf welche Art und Weise in ihr gehandelt werden soll." ${ }^{23}$ Diese Gegenüberstellung von Erkenntnis und Wahrheit vs. Urteilen und Entscheiden verweist darauf, dass das Politische eine bestimmte Dimension der „reinen” Entscheidung darstellt: es gibt ein Moment der Wahl, das durch die Kenntnis der Voraussetzungen und Folgen einer Entscheidung nicht ersetzt werden kann. So ist ihr Begriff von der Politik eine Kritik an der Reduktion von den politischen Fragen auf die technischen. Arendt denkt das weiter im Sinne einer radikalen Maßstabslosigkeit des politischen Handelns überhaupt. Es gibt nirgendwo einen Punkt, an dem ein nichtpolitisches Kriterium der politischen Entscheidung Grenzen setzt, an denen sie sich ausrichten muss. In diesem Sinne ist der eigentlich politische Bereich „maßstabslos”: in ihm gibt es keine „richtigen” Lösungen in dem Sinne, dass deren Kriterien außerhalb des politischen Prozesses (dem "gemeinsamen Begutachten, Bereden und Entscheiden") selbst liegen. Die Maßstäbe, nach denen geurteilt wird, werden im Prozeß des Urteilens erst generiert. Die Politik bezeichnet so einen Bereich von prinzipiell offener Auseinandersetzungen, die weder im Bezug auf Wahrheit noch auf Tradition zu lösen sind.

Die Maßstablosigkeit entspricht Arendts Assoziation des Politischen mit der Fähigkeit, etwas neu zu beginnen. Es sei „[...] die Funktion jeden Handelns, im Unterschied zu einem bloß reaktiven Sichverhalten (behavior), Prozesse zu unterbrechen, die sonst automatisch und damit vorhersagbar

${ }^{23}$ H. Arendt, Zwischen Vergangenheit und Zukunft, München 1994, S. 300. 
verlaufen würden"24. Politisches Handeln also ist bei Arendt ein wesentlich innovatives Handeln und deswegen darf es sich auf keine festgelegten Maßstäbe berufen. Arendt bezieht sich in diesem Zusammenhang sogar auf den religiösen Terminus „Wunder”, der für das Unvordenkliche, nicht Erwartbare steht: „Wenn der Sinn von Politik Freiheit ist, so heißt dies, daß wir in diesem Raum - und in keinem andern - in der Tat das Recht haben, Wunder zu erwarten” ${ }^{25}$. Der Bereich der „Wunder” ist aber nicht der des Alltags. Hier liegt der Einwand nahe, dass die Politik nicht nur aus neuen Ideen und neuen Problemen besteht, es darüber hinaus einen „interessenfreien Raum” nahezu (mit Ausnahme von Revolutionen) nie gibt und zum Beispiel die Aspekte gerechter Verteilung von Gütern, die prinzipiell begrenzt sind, immer zur Politik gehören. Wenn solch eine kritische Interpretation zutreffen würde, würde dies bedeuten, dass Arendts Begriff des Politischen paradoxerweise zur Entpolitisierung der Politik führt, wie das bemerkenswerte Buch von Dana Vila über Arendt und Heidegger zeigt ${ }^{26}$. Arendts Begriff des Politischen hätte dann keinen nachvollziehbaren Bezug mehr auf Erfahrungen und Möglichkeiten des Politischen im Alltag komplexer demokratischer Gesellschaften: Politik würde zu etwas anderem werden als das Politische, wie wir es heutzutage kennen. Es scheint aber, dass es Arendt eher um ein Verständnis von Politik als einem Raum von Möglichkeiten geht, der die „Normalität” (also die institutionelle Ordnung) bildet.

Es bleibt sowieso unklar, ob in ihrem Verständnis vom politischen Handeln das „authentische” Handeln zu Grunde liegt, es als Modus kollektiver Selbstbestimmung verstanden werden kann, oder ob es um „eine radikale Wahl” geht, die wie bei Schmitt normativ „aus dem Nichts” kommt. Die Frage ist also: Handelt es sich im Politischen um begründete und begründbare Entscheidungen? oder anders gesagt: beruht das „,[...] urteilende Begutachten und Bereden der gemeinsamen Welt und die Entscheidung darüber, wie sie weiterhin aussehen und auf welche Art und Weise in ihr gehandelt werden soll" 27 auf einer rationalen Wahl und teilbaren Überlegungen, oder geht es hier um irrationale Wahl? Im letzten Fall bekäme der Neuanfang, auf den Arendt zielt, einen absurden Sinn.

\footnotetext{
${ }^{24}$ H. Arendt, Macht und Gewalt, München 1987, S. 35.

${ }^{25}$ H. Arendt, Was ist Politik?, München 1993, S. 35.

${ }^{26}$ D. Vila, Arendt and Heidegger - The Fate of the Political, Princeton 1996.

${ }^{27}$ H. Arendt, Zwischen Vergangenheit..., op. cit., S. 300.
} 
Wenn ich eine Wahl treffe und keine Gründe dafür dabei in Betracht ziehe, überlasse ich mich einfach dem Zufall - insofern man sagen kann: es war nicht ich, der gewählt hat. Noch deutlicher wird der Widersinn eines solchen Verständnisses des politischen Handelns, wenn man sich vor Augen führt, dass es Arendt um ein gemeinsames Handeln geht. Gerade dann, wenn die Entscheidungen gemeinsam getroffen werden sollen, muss argumentiert werden, damit andere überzeugt werden. Das Verständnis des Politischen als Selbstregierung der gemeinsam Sprechenden und Handelnden erfordert also eine Orientierung an rationaler Begründung. Auch wenn es im Politischen keine „letzten Gründe” gibt, so gibt es doch den Sinn von Begründungen. Im Politischen geht es nicht nur um revolutionäre, "rauschhafte” Augenblicke, sondern auch um gute, intersubjektiv teilbare Gründe.

Es lässt sich nicht eindeutig entscheiden, welche der oben diskutierten Bedeutungen der "politischen Wahl” der theoretischen Absicht Arendts entspricht, weil wir in ihren Texten Formulierungen finden können, die eine oder andere Interpretation zu beweisen scheinen. Ganz sicher schlägt Arendt keine irrationale Vision des Politischen vor, obwohl auch die dezisionistischen Motive eine Rolle in ihrer „Revolutionsromantik” spielen. Der Anspruch ihrer politischen Philosophie bleibt aus dem Grund rational, wie sich nicht zuletzt aus ihrer affirmativen Bezugnahme auf die Constitutio Libertatis in der amerikanischen Revolution ergibt. Daraus ergibt sich die Schlussfolgerung, dass Arendt eine zu starke Neigung - vor allem im Revolutionsbuch - zeigte, die Rolle von Begründungen im Bereich des Politischen zu unterschätzen. Der problematische Charakter der Arendtschen Konzeption liegt überdies in der Dichotomie zwischen dem bloßen, nichtpolitischen und dem "guten", politischen Leben. Das ist der Preis, welchen ihr Begriff von Politik für seine Herleitung aus dem Modell der Polis zahlt. Arendt sympathisiert hier augenfällig mit der konservativen Tradition (Schmitt, Jünger), welche die Gesellschaft als eine „dunkle” Sphäre niederer Tätigkeiten gegenüber der „hellen”, erhabenen Sphäre des reinen darstellt und zu einem interessenlosen politischen Handeln abwertet. Solch eine scharfe Trennung führt aber nicht nur zu einer völlig inadäquaten Einschätzung der Aufgaben moderner Staaten, sie erlaubt es auch nicht, die Dynamik sozialer Bewegungen zu verstehen.

In einem empfehlenswerten Artikel betont Seyla Benhahib, dass heutzutage die sozialen Fragen - aber auch der „Kampf um Anerkennung”28 verschiedener Gruppen im Bereich des Rechts, der personalen und kollektiven

${ }^{28}$ Vgl. A. Honneth, Kampf um Anerkennung, Frankfurt am Main 1992. 
Identität, sozialer Wertschätzung etc. - im Öffentlichmachen des „nur” Privaten besteht. Soziale Themen drängen sich also - metaphorisch gesagt - aus dem „Dunkel” des Haushalts in die „Helle der Öffentlichkeit”, und deswegen beruht die Politik darauf, diese dunklen Bedürfnisse in rationale, diskutierbare Ansprüche zu verwandeln. „Nach der französischen und amerikanischen Revolution erweitert sich - schreibt Benhahib - der Umfang des Öffentlichen mit dem Eintritt jeder neuen Gruppe in den öffentlichen Raum der Politik. Die Emanzipation der Arbeiter hat die Eigentumsverhältnisse zu einer öffentlich-politischen Streitfrage gemacht; die Emanzipation der Frauen bedeutet, daß die Familie und die sogenannte Privatsphäre zu politischen Fragen geworden sind; die Erlangung von Rechten durch Nicht-Weiße und Nicht-Christen hat kulturelle Fragen der kollektiven Selbst- und Fremdrepräsentation auf die "öffentliche" Tagesordnung gesetzt" ${ }^{29}$. Benhahib schließt daraus: „Die Unterscheidung zwischen dem »Gesellschaftlichen« und dem "Politischen « ist in der modernen Welt nicht sinnvoll, und zwar nicht wie Hannah Arendt dachte, weil alle Politik zur Verwaltung und Ökonomie das wesentlich „Öffentliche”, sondern vor allem, weil der Kampf, etwas öffentlich zu machen, ein Kampf um Gerechtigkeit geworden ist"30.

\section{Schlussbemerkung - Arendt und die liberale Demokratie}

Wir hatten bereits darauf hingewiesen, dass Arendt zwar den Kantischen Universalismus der Menschen- und Bürgerrechte akzeptiert, aber auch beide Arten von Rechte scharf trennt. Sie deutet die Idee der Menschenrechte als eine bloß moralische Idee, das heißt vorpolitisch, und die Idee der Bürgerrechte im Sinne einer bloßen Rechtstaatsidee. Bürgerrechte sind immer nur Rechte derer, die zur bestimmten politischen Gemeinschaft gehören, deshalb ist es kein Zufall, dass die Verwirklichung der Menschen- als Bürgerrechte in der französischen Revolution zugleich die Geburtsstunde des europäischen Nationalstaates war. Menschenrechte, Bürgerrechte, Gerechtigkeit sind Zentralbegriffe, die die Grammatik des liberal-demokratischen Diskurses bestimmen. Arendt wertet aber diese Kategorien ab, deswegen tut sich in ihrem Ansatz die Kluft zwischen der Ebene der Grundrechte und der Idee politischer Freiheit auf. Ihr Hauptthema ist demnach nicht Gerechtigkeit, sondern

${ }^{29}$ S. Benhahib, Modelle des öffentlichen Raums: Hannah Arendt, die liberale Tradition, Jürgen Habermas [in:] „Soziale Welt” (42), 1991, S. 152.

${ }^{30}$ Ibidem. 
die (politische) Freiheit. Die „echte’ Politik entwickelt sich gewissermaßen von Natur aus inmitten kontingenter geschichtlicher Umstände in einem „Raum”, der keinem teleologischen Gesetz unterliegt und durch kein normatives Fundament abgesichert und begründet ist. Die Möglichkeit politischer Freiheit ist jedoch universal, insofern sie aus den „Elementarbedingungen des Handelns" folgt.

Jede tatsächliche Konstitution eines Raums öffentlicher Freiheit kann dagegen nur die Konstitution eines begrenzten "gezäunten" - wie Arendt schreibt - Freiheitsraumes sein, weil sie nur aus dem kontingenten und immer sozusagen lokalen Akt der „revolutionären Tat” einer bestimmter Gruppe zusammenhandelnder Menschen resultieren kann. Institutionen der Freiheit müssen erfunden werden, ihre Etablierung muss gelingen (und kann mehr oder weniger mißlingen), ihre Bewahrung bedeutet im gewissen Sinne ihre beständige Neuerfindung, Neukonstituierung. Sie bringen jedesmal neue Erfahrungen und neue Einstellungen hervor.

Das Kriterium „öffentlicher Freiheit”, das die „direkte” von der bloß repräsentativen Demokratie unterscheidet, lässt sich, Arendt nach, aus einem Prinzip gleicher demokratischer Grundrechte nicht ableiten - und mehr noch - es gibt eigentlich kein universelles Prinzip (wie die Forderung allgemeiner und gleicher Rechte), aufgrund dessen man Institutionen politischer Freiheit aufbauen könnte. Die Formen, in denen sie Realität gewinnen, hängen wesentlich von verschiedenen unvorhersehbaren Umständen, von kulturellen Traditionen, von materiellen Bedingungen ebenso wie von der Initiative, der Phantasie, der Entschlossenheit und der Urteilskraft der Individuen ab. Kurz gesagt: die politische Freiheit ist einerseits ein überall mögliches und gleichsam "natürliches” Projekt der Menschen, andererseits hat sie immer einen wesentlich „lokalen” und partikularen Charakter.

Die Herausforderung, die Arendts Denken für die gegenwärtige politische Philosophie von Bedeutung zu sein scheint, besteht darin, dass das politische Denken in der Tradition der liberalen und sozialen Demokratie kaum Kategorien ausgebildet hat, in welchen sich der - für die liberale Demokratie selbst essentielle - Bereich öffentlicher Freiheit angemessen fassen lässt. Zum Beispiel, John Rawls, einer der wichtigsten gegenwärtigen Demokratietheoretikern stellt sich die öffentliche Autonomie als passive und aktive Stimmrechte, als demokratisches Entscheidungsverfahren etc., in solchen Begriffen also vor, die für die konstruktive Aneignung oder für die eventuelle Reformulierung von Arendts Ideen einer „direkten” Demokratie, eines Zusammenhandeln Freier und Gleicher sicherlich unzureichend sind. Auch 
die kommunitarischen Theoretiker, die Arendts republikanischen Ideen näher stehen als Rawls, sind unempfänglich für ihren Radikalismus. Die Philosophin weist alle Träume von Gemeinschaft und gemeinschaftstiftenden Werten - seien sie nationaler, religiöser oder gar ethnischer Art - zurück. Sie ist eher an dem Potential des Neuen in der republikanischen Freiheit interessiert - also an der Eröffnung einer gemeinsamen Welt und nicht an der Rückkehr zu einer vergangenen. Insofern dachte sie - so kann man sagen wahrhaft revolutionär.

Andererseits scheint ihre scharfe Entgegensetzung von repräsentativer und direkter Demokratie problematisch, wenn nicht sogar naiv zu sein. Wir haben darüber schon im vorigen Abschnitt geschrieben. Die zentralen Kategorien, durch die sie die Idee öffentlicher Freiheit erläutert, lassen sich weder in einem normativen noch in einem funktionalen Sinn auf moralische, soziale Kategorien oder Rechtskategorien zurückzuführen. Zugleich trennt sie entschieden die spezifisch politischen Probleme von solchen, die zur Sphäre der Moral, der sozialen Wohlfahrt, der Privatsphäre, der Ökonomie, aber auch des Schutzes von den Grundrechten gehören. Dies ist zweifelsohne die wichtigste Schwäche ihres Ansatzes und der Grund dafür, dass ihre Ideen heutzutage gleichsam brachliegen. Irritierend für gegenwärtige Leser kann auch Arendts Neigung sein, den Rätegedanken am Ende des Revolutionsbuches wörtlich zu nehmen. Sie will nämlich die Räte von all den sozialen, administrativen und ökonomischen Problemen entlasten, mit denen sie sich - wie die Autorin beklagt - in den Augenblicken der Revolution immer belastet hatten.

Jeden Tag erfahren wir am eigenen Leibe, dass die Sphäre des Politischen den Problemen der sozialen Gerechtigkeit, der Ökonomie etc. nicht einfach den Rücken zukehren kann. Im Falle der Grundrechte oder der sozialen Gerechtigkeit handelt es sich um "gemeinsame Angelegenheiten" jener Art, wie sie nach Arendt in den republikanischen Institutionen verhandelt werden sollten. Sobald man dies aber zugesteht, wird deutlich, dass die individuelle Freiheit, soziale Gerechtigkeit und öffentliche Freiheit eng miteinander verbunden sind.

\section{Literaturverzeichnis}

Arendt, H. Über die Revolution, München 1974.

Arendt, H. Vita activa oder vom tätigen Leben, München 1981. 
Arendt, H. Zwischen Vergangenheit und Zukunft, München 1994.

Arendt, H. Macht und Gewalt, München 1987.

Arendt, H. Was ist Politik?, München 1993.

Benhahib, S. Modelle des öffentlichen Raums: Hannah Arendt, die liberale Tradition, Jürgen Habermas (in:) „Soziale Welt” (42), 1991.

Honneth, A. Kampf um Anerkennung, Frankfurt am Main 1992.

Jaeggi, R. Welt und Person. Zum anthropologischen Hintergrund der Gesellschaftskritik Hannah Arendts, Berlin 1997.

Vila, D. Arendt and Heidegger - The Fate of the Political, Princeton 1996.

Żiżek, S. Die Revolution steht bevor. Dreizehn Versuche über Lenin, übers. von N. G. Schneider, Franfurt am Main 2002.

\section{Abstract \\ Comments on Hannah Arendt's Theory of Revolution}

This article contains a critical commentary on Hannah Arendt's theory of revolution. The first part (the first two paragraphs) discusses the main categories by which the philosopher defines the essence of every revolution, namely politics, public freedom, direct democracy, action as a praxis, council system, novum, and pluralism. In the second part of the article argumentative deficiencies are indicated, which come down to two points: a hidden irrationality of the notion of "the political" and the excessive sharpness of the opposition between direct and parliamentary democracy.

Key words: revolution, Hannah Arendt, politics, public freedom, direct democracy, the action, council system, novum, pluralism 